\title{
Male Circumcision for the Prevention of HSV-2 and HPV Infections and Syphilis
}

\author{
Aaron A.R. Tobian, M.D., Ph.D ${ }^{1}$, David Serwadda, M.Med., M.P.H ${ }^{6}$, Thomas C. Quinn, M.D., \\ M.Sc ${ }^{2,8}$, Godfrey Kigozi, M.B., Ch.B., M.P.H ${ }^{7}$, Patti E. Gravitt, Ph.D ${ }^{3}$, Oliver Laeyendecker, \\ M.S., M.B.A ${ }^{2,8}$, Blake Charvat, M.Sc ${ }^{4}{ }^{\star}$, Victor Ssempijja, B.Stat ${ }^{7}$, Melissa Riedesel, M.P.H ${ }^{8}$, \\ Amy E. Oliver, B.A ${ }^{2}$, Rebecca G. Nowak, M.P. $H^{3}$, Lawrence H. Moulton, Ph. $D^{5}$, Michael $Z$. \\ Chen, M.Sc ${ }^{4}$, Steven J. Reynolds, M.D., M.P.H ${ }^{2}, 8^{2}$, Maria J. Wawer, M.D., M.H.Sc ${ }^{4}$, and Ronald \\ H. Gray, M.D., M.Sc. ${ }^{4}$ \\ ${ }^{1}$ Department of Pathology, Johns Hopkins University, Baltimore ${ }^{2}$ Department of Medicine, Johns Hopkins \\ University, Baltimore ${ }^{3}$ School of Medicine, and the Departments of Epidemiology and Molecular \\ Microbiology and Immunology, Johns Hopkins University, Baltimore 4 Population, Family, and Reproductive \\ Health, Johns Hopkins University, Baltimore ${ }^{5}$ International Health and Biostatistics, Johns Hopkins \\ University, Baltimore ${ }^{6}$ Bloomberg School of Public Health, Johns Hopkins University, Baltimore; the \\ Institute of Public Health, Makerere University, Kampala 7 Rakai Health Sciences Program, Entebbe - both \\ in Uganda 8 Division of Intramural Research, National Institute of Allergy and Infectious Diseases, National \\ Institutes of Health, Bethesda, MD
}

\section{Abstract}

BACKGROUND-Male circumcision significantly reduced the incidence of human immunodeficiency virus (HIV) infection among men in three clinical trials. We assessed the efficacy of male circumcision for the prevention of herpes simplex virus type 2 (HSV-2) and human papillomavirus (HPV) infections and syphilis in HIV-negative adolescent boys and men.

METHODS-We enrolled 5534 HIV-negative, uncircumcised male subjects between the ages of 15 and 49 years in two trials of male circumcision for the prevention of HIV and other sexually transmitted infections. Of these subjects, 3393 (61.3\%) were HSV-2-seronegative at enrollment. Of the seronegative subjects, 1684 had been randomly assigned to undergo immediate circumcision (intervention group) and 1709 to undergo circumcision after 24 months (control group). At baseline and at 6,12, and 24 months, we tested subjects for HSV-2 and HIV infection and syphilis, along with performing physical examinations and conducting interviews. In addition, we evaluated a subgroup of subjects for HPV infection at baseline and at 24 months.

RESULTS-At 24 months, the cumulative probability of HSV-2 seroconversion was $7.8 \%$ in the intervention group and $10.3 \%$ in the control group (adjusted hazard ratio in the intervention group, $0.72 ; 95 \%$ confidence interval $[\mathrm{CI}], 0.56$ to $0.92 ; \mathrm{P}=0.008$ ). The prevalence of high-risk HPV genotypes was $18.0 \%$ in the intervention group and $27.9 \%$ in the control group (adjusted risk ratio, $0.65 ; 95 \% \mathrm{CI}, 0.46$ to $0.90 ; \mathrm{P}=0.009$ ). However, no significant difference between the two study groups was observed in the incidence of syphilis (adjusted hazard ratio, 1.10; 95\% CI, 0.75 to 1.65; $\mathrm{P}=0.44)$.

Address reprint requests to Dr. Quinn at the Rangos Building, Rm. 530, 855 N. Wolfe St., Baltimore, MD 21205, or at E-mail: tquinn@jhmi.edu.

Drs. Quinn and Gray contributed equally to this article.

*Deceased.

This study is dedicated to the memory of Blake Charvat, who died on August 21, 2008. 
CONCLUSIONS-In addition to decreasing the incidence of HIV infection, male circumcision significantly reduced the incidence of HSV-2 infection and the prevalence of HPV infection, findings that underscore the potential public health benefits of the procedure.

Herpes simplex virus type 2 (HSV-2) and human papillomavirus (HPV) infections and syphilis are common sexually transmitted infections. HSV-2 infection and syphilis are two of the main causes of genital ulceration ${ }^{1-3}$ and have been associated with an increased risk of human immunodeficiency virus (HIV) infection in observational studies. ${ }^{1,2,4}$ The prevalence of HPV is significantly increased in developing nations. ${ }^{5} \mathrm{HPV}$ infection can cause genital warts, and high-risk HPV genotypes are associated with penile and anal cancer, as well as with cervical cancer in female partners. 5,6

Three randomized trials and multiple observational studies showed that male circumcision significantly decreased the incidence of HIV infection in male subjects. ${ }^{7-9}$ The rate of selfreported genital ulcer disease was decreased among circumcised men in a trial conducted in Rakai, Uganda, which suggested that male circumcision might reduce the incidence and prevalence of ulcerative sexually transmitted infections. ${ }^{7}$ However, previous findings with regard to the effects of male circumcision on the incidence of HSV-2 infection and syphilis have been more equivocal. Two observational studies suggested that male circumcision significantly decreased the incidence of HSV-2 infection, 10,11 whereas other studies showed no association. ${ }^{12-15}$ Similarly, two observational studies showed that male circumcision decreased the incidence of syphilis, ${ }^{16,17}$ whereas other studies showed no association. ${ }^{15,18}$ According to a meta-analysis of circumcision studies, among male subjects who had been circumcised, the odds ratio for HSV-2 infection was 0.88 (95\% confidence interval [CI], 0.77 to 1.01 ), and the odds ratio for syphilis was 0.67 (95\% CI, 0.54 to 0.83 ), as compared with those who had not been circumcised. ${ }^{19}$ Although most observational studies have suggested that male circumcision decreases the incidence of penile HPV infection, ${ }^{20,21}$ some studies have shown no effect. 22,23

Many of the observational studies evaluating male circumcision and sexually transmitted infections had limited statistical power, were vulnerable to confounding by sexual practices correlated with a high risk of transmission, and evaluated the status of circumcision solely on the basis of self-report. Thus, the potential efficacy of male circumcision for the prevention of sexually transmitted infections can be determined only in randomized trials. We used data from randomized, controlled trials of male circumcision for the prevention of HIV infection in Rakai, Uganda, to assess the efficacy of male circumcision for the prevention of HSV-2 and HPV infections and syphilis in adolescent boys and men.

\section{METHODS}

\section{STUDY DESIGN AND SUBJECTS}

We conducted two parallel but independent trials of male circumcision for the prevention of HIV infection and other sexually transmitted infections in Rakai, Uganda, as described previously. 7,24 In the first trial (Rakai-1, which was funded by the National Institutes of Health), we enrolled 4996 HIV-negative, uncircumcised boys and men between the ages of 15 and 49 years who accepted voluntary HIV counseling and testing and agreed to learn their HIV results. The second trial (Rakai-2, which was funded by the Bill and Me-linda Gates Foundation) had as its primary goal the assessment of the safety of male circumcision and its effects on sexually transmitted infections in HIV-infected men and their partners. However, the latter trial also included 595 HIV-negative subjects, of whom 155 (26.1\%) declined to learn their HIV results and 440 (73.9\%) agreed to learn their HIV results but were included in the study to provide blinding of subjects' HIV status so as to prevent potential stigmatization of HIV-infected subjects who were participating in the trial. 
The two trials, which were conducted concurrently, had identical protocols. At each visit, all subjects were offered free HIV counseling and testing, health education, and condoms. All subjects who were found to be HIV-positive were referred to an HIV treatment program funded by the U.S. President's Emergency Plan for AIDS Relief (PEPFAR). All subjects provided written informed consent.

Both the Rakai-1 and Rakai-2 trials were approved by four institutional review boards: the Science and Ethics Committee of the Uganda Virus Research Institute (Entebbe, Uganda), the HIV subcommittee of the National Council for Science and Technology (Kampala, Uganda), the Committee for Human Research at Johns Hopkins University's Bloomberg School of Public Health (Baltimore, MD), and the Western Institutional Review Board (Olympia, WA). The Rakai-1 trial was overseen by the data and safety monitoring board for vaccine safety and prevention of the National Institutes of Health and by a separate board for Rakai-2.

We performed physical examinations and conducted interviews to ascertain sociodemographic characteristics and rates of sexual practices at baseline and at 6, 12, and 24 months. Additional details on the study design, specimen testing, and analyses are presented in the Supplementary Appendix, available with the full text of this article at NEJM.org.

\section{VIRUS AND SYPHILIS DETECTION}

HSV-2 testing was performed with the use of an enzyme-linked immunosorbent assay (ELISA) (Kalon Biological). On the basis of previous evaluation of test performance in Ugandan serum samples, subjects who had positive tests for HSV-2 had an optical-density index value of 1.5 or more, ${ }^{25}$ and all seroconversions that were detected by ELISA were confirmed by Western blot (Euroimmun). HIV status was determined with the use of two separate ELISAs and confirmed by HIV-1 Western blot analysis, as described previously. ${ }^{7}$

HPV genotyping was performed with the use of the HPV Linear Array (Roche Diagnostics), as described previously. ${ }^{26,27} \mathrm{HPV}$ genotypes $16,18,31,33,35,39,45,51,52,56,58,59,66$, and 68 were considered the primary high-risk (carcinogenic) genotypes. ${ }^{28,29}$ (We included HPV genotype 66 [HPV-66] in our definition of carcinogenic HPV genotypes after its reclassification, in $2005 .{ }^{29}$ ) For the primary analyses of HPV prevalence, we excluded subjects who were HPV-negative and had no detectable beta-globin in the sample, since the presence of cellular material could not be demonstrated.

Active Treponema pallidum infection was determined by means of a positive rapid plasma reagin test (Becton Dickinson) or a toluidine red unheated serum test (TRUST) (New Horizons Diagnostics) and was confirmed by a positive T. pallidum particle agglutination assay (Serodia TP-PA kit, Fujirebio).

\section{STATISTICAL ANALYSIS}

For end points for HSV-2 infection and syphilis, we performed time-to-event analyses, using the protocol-specified Kaplan-Meier method, with the end point defined as the time to the detection of HSV-2 or syphilis seropositivity, with censoring of data at the last visit. In the primary intention-to-treat analysis, we performed an adjustment for baseline characteristics using a Cox proportional-hazards model for the time to detection of HSV-2 or syphilis seropositivity. In secondary analyses, adjustments were also made for the trial (Rakai-1 or Rakai-2), changes in sexual practices (number of sexual partners, condom use, and alcohol use with sexual intercourse), and symptoms of sexually transmitted infections (genital ulcers, urethral discharge, and dysuria) during follow-up as time-varying covariates. In addition, we conducted an as-treated analysis in which crossover subjects (i.e., subjects in the intervention 
group who did not undergo surgery and those in the control group who underwent surgery elsewhere) were classified according to their actual circumcision status.

We used Poisson regression to estimate incidencerate ratios, risk ratios for prevalence, and 95\% confidence intervals. Additional details regarding subgroup analyses of the incidence of HSV-2 infection according to covariates and HPV analysis are available in the Supplementary Appendix. The rates of sexual practices and symptoms of sexually transmitted infections were tabulated at each follow-up visit, and differences between the two study groups were assessed with the use of chi-square tests. All reported P values are two-sided and have not been adjusted for multiple testing.

\section{RESULTS SUBJECTS}

Subjects were enrolled in the Rakai-1 trial from September 2003 through September 2005 and in the Raiki-2 trial from February 2004 through December 2006. Of the 6396 subjects who were initially screened in both the Rakai- 1 and Rakai- 2 trials, 3003 were excluded from the analyses reported here because of preexisting positive or indeterminate HSV-2 or HIV-1 status (Fig. 1). For the analysis to determine HSV-2 seroconversion, we evaluated 3393 HIVnegative, HSV-2-negative, uncircumcised subjects between the ages of 15 and 49 years; of these subjects, 1684 had been randomly assigned to undergo immediate circumcision (intervention group) and 1709 to undergo circumcision in 24 months (control group). The retention rates at 24 months were $81.9 \%$ (1370 of 1673 subjects) in the intervention group and $82.0 \%$ (1395 of 1701) subjects in the control group.

For the HSV-2 study population, baseline sociodemographic characteristics and rates of sexual practices and symptoms of sexually transmitted infections were similar in the two study groups (Table 1). Subjects who were enrolled in the Rakai-2 trial had higher sexual-risk profiles at enrollment than subjects in the Rakai-1 trial because the Rakai-2 trial permitted enrollment of subjects who declined to learn their HIV status, whereas the Rakai-1 trial required receipt of HIV results. The Rakai-2 subjects were significantly older than those in the Rakai-1 trial, were more likely to be currently or previously married, had had a higher number of sexual partners in the previous year, and had had a higher rate of alcohol use with sex in the previous 6 months.

\section{MALE CIRCUMCISION AND HSV-2 ACQUISITION}

At 24 months in the intention-to-treat population, HSV-2 infection was detected in 114 subjects in the intervention group and in 153 subjects in the control group (Fig. 2). The cumulative probability of HSV-2 infection during the 24-month period was lower in the intervention group $(7.8 \%)$ than in the control group $(10.3 \%)$ with an unadjusted hazard ratio of 0.75 (95\% CI, 0.60 to $0.94 ; \mathrm{P}=0.02$ ). After adjustment for enrollment characteristics and rates of sexual practices and symptoms of sexually transmitted infections, the hazard ratio was 0.72 (95\% CI, 0.56 to $0.92 ; \mathrm{P}=0.008)$. After adjustment for time-varying covariates during follow-up, the hazard ratio was $0.77(95 \% \mathrm{CI}, 0.62$ to $0.97 ; \mathrm{P}=0.03)$. In the as-treated analysis, the unadjusted hazard ratio for detection of infection was $0.73(95 \% \mathrm{CI}, 0.59$ to $0.93 ; \mathrm{P}=0.01)$; after adjustment for baseline characteristics, the hazard ratio was $0.72(95 \% \mathrm{CI}, 0.59$ to $0.91 ; \mathrm{P}=0.009)$.

In separate analyses of data from the Rakai- 1 and Rakai- 2 trials, male circumcision reduced the incidence of HSV-2 infection in both trials. In the Rakai- 1 trial, the cumulative probability of HSV-2 infection at 24 months in the intention-to-treat population was lower in the intervention group (7.7\%) than in the control group $(9.9 \%)$, with an unadjusted hazard ratio of 0.77 ( $95 \%$ CI, 0.59 to 0.99 ). In the Rakai-2 trial, the cumulative probability of HSV-2 infection was $8.6 \%$ in the intervention group and $14.0 \%$ in the control group (unadjusted hazard ratio, 
$0.59 ; 95 \% \mathrm{CI}, 0.27$ to 1.27$)$. There was no significant difference in efficacy between the Rakai-1 trial and the Rakai-2 trial ( $\mathrm{P}=0.52$ for interaction).

The cumulative rates of HSV-2 seroconversion per 100 person-years, sexual practices, and symptoms of sexually transmitted infections are shown in Figure 3. Overall, the incidence of HSV-2 infection was lower among circumcised subjects, and there were no significant differences in the hazard ratios in subgroup analyses. Subjects who reported symptoms of sexually transmitted infections had a higher incidence of HSV-2 infection than asymptomatic subjects. At 24 months, the cumulative prevalence rates of self-reported symptoms of genital ulcer disease were higher among subjects with HSV-2 seroconversion (10.3\%) than among subjects without serocon-version (2.7\%) (relative risk, 3.78; 95\% CI, 2.87 to 4.98 ; $\mathrm{P}<0.001$ ).

For the HSV-2 study population, rates of sexual practices that were stratified according to circumcision status are shown in Table 2. At 6 months, reported condom use was higher in the intervention group than in the control group $(\mathrm{P}<0.001)$, but no significant differences between the two study groups were observed thereafter. There were no significant between-group differences in the reported number of sexual partners, but nonmarital sexual relationships were more frequently reported by subjects in the intervention group than in the control group, and these differences were significant at 12 and 24 months $(P=0.04$ and $\mathrm{P}=0.03$, respectively). At all follow-up intervals, rates of reported alcohol use with sexual intercourse were higher in the control group than in the intervention group, differences that were significant at 6,12 , and 24 months. Reported transactional sexual intercourse (which was defined as the exchange of sex for money or gifts) was infrequent, and rates did not differ significantly between the two study groups. Genital ulcer disease was more frequent in the control group than in the intervention group at 6 months $(\mathrm{P}<0.001), 12$ months $(\mathrm{P}<0.001)$, and 24 months $(\mathrm{P}=0.02)$. However, there were no significant differences between the two groups in reported genital discharge or dysuria.

\section{MALE CIRCUMCISION AND ACQUISITION OF SYPHILIS}

To determine the efficacy of circumcision in preventing syphilis, subjects who tested negative for HIV and T. pallidum infections at baseline were evaluated for active T. pallidum infection during follow-up. Enrollment characteristics were similar in the two study groups. At 24 months, syphilis was detected in 50 of 2083 subjects (2.4\%) in the intervention group, as compared with 45 of 2143 subjects $(2.1 \%)$ in the control group (hazard ratio, 1.14; 95\% CI, 0.77 to $1.75 ; \mathrm{P}=0.50$ ). Adjustment for enrollment characteristics and rates of sexual practices and symptoms of sexually transmitted infections did not significantly affect these estimates of circumcision efficacy (adjusted hazard ratio, $1.10 ; 95 \% \mathrm{CI}, 0.75$ to $1.65 ; \mathrm{P}=0.44$ ).

\section{MALE CIRCUMCISION AND PREVALENCE OF HPV}

Overall, baseline sociodemographic characteristics and rates of sexual practices and symptoms of sexually transmitted infections were similar in the two study groups for subjects in the HPV sub-study population (see the table in the Supplementary Appendix). At enrollment, the prevalence of high-risk HPV genotypes was $38.1 \%$ in the intervention group and $37.1 \%$ in the control group $(\mathrm{P}=0.79)$.

In the primary intention-to-treat analyses at 24 months, high-risk HPV genotypes were detected in 42 of 233 subjects in the intervention group (18.0\%), as compared with 80 of 287 subjects in the control group (27.9\%), with an unadjusted risk ratio of 0.65 (95\% CI, 0.45 to $0.94 ; \mathrm{P}=$ 0.01 ) (Table 3). Adjustment for enrollment characteristics and rates of sexual practices and symptoms of sexually transmitted infections did not significantly affect this estimate (adjusted risk ratio, $0.65 ; 95 \% \mathrm{CI}, 0.46$ to $0.90 ; \mathrm{P}=0.009$ ). When the analysis was confined to subjects who had beta-globin-positive samples at the 24-month visit, high-risk HPV genotypes were 
detected in 32 of 215 subjects (14.9\%) in the intervention group, as compared with 69 of 260 subjects (26.5\%) in the control group (risk ratio, 0.56 ; $95 \% \mathrm{CI}, 0.37$ to $0.85 ; \mathrm{P}=0.007$ ). At 24 months, multiple high-risk HPV genotypes were detected in 10 of 233 subjects $(4.3 \%)$ in the intervention group and in 35 of 287 subjects (12.2\%) in the control group (risk ratio, 0.35; 95\% $\mathrm{CI}, 0.17$ to $0.71 ; \mathrm{P}=0.004)$. The prevalence of non-high-risk HPV genotypes at the 24-month visit was also lower in the intervention group (26.2\%) than in the control group (39.4\%) (risk ratio, $0.66 ; 95 \% \mathrm{CI}, 0.49$ to $0.91 ; \mathrm{P}=0.01$ ).

\section{DISCUSSION}

In our study, the circumcision of adolescent boys and men in a rural Ugandan population significantly reduced the incidence of HSV-2 infection and the prevalence of HPV infection during 24 months of follow-up but did not affect the incidence of syphilis. The efficacies of circumcision for the prevention of HSV-2 incidence and HPV prevalence were $25 \%$ and $35 \%$, respectively. Adjustment resulted in a modest increase in efficacy to $28 \%$ for HSV-2 infection but did not change efficacy for HPV infection. These findings are compatible with those of observational studies, in which reduced rates of HSV-2 and HPV infections were associated with circumcision, $10,11,19-21$ and with the results of one clinical trial, which showed that circumcision decreased the risk of HPV infection. ${ }^{30,31}$ These findings, in conjunction with those of previous trials, indicate that circumcision should now be accepted as an efficacious intervention for reducing heterosexually acquired infections with HSV-2, HPV, and HIV in adolescent boys and men. However, it must be emphasized that protection was only partial, and it is critical to promote the practice of safe sex.

The biologic mechanisms for the reduction in rates of HSV-2 and HPV infections by means of circumcision may involve anatomical factors, cellular factors, or both. The retraction of the foreskin over the shaft during intercourse exposes the inner preputial mucosa to vaginal and cervical fluids 7,19,32 and can also result in microtears during intercourse, particularly in the frenulum. 7,19,32 The moist subpreputial cavity may provide a favorable environment for the survival of HSV-2 and HPV and consequent epithelial infection. 7, 19,33 Both HSV-2 and HPV replicate in epithelial cells of the epidermis and dermis, ${ }^{34}$ and the inner mucosa of the foreskin is lightly keratinized, which may facilitate the access of HSV-2 and HPV to underlying epithelial cells in uncircumcised adolescent boys and men. After circumcision and keratinization of the surgical scar, the risk of such epithelial infection is probably reduced.

In observational studies, 2,35 genital HSV-2 infection has been associated with an increased risk of HIV acquisition. In our trial, we found that male circumcision reduced the rate of symptomatic genital ulcerative disease. ${ }^{7}$ Since genital ulcerative disease and HSV-2 infection are both thought to be cofactors in HIV acquisition, 2,36,37 male circumcision may provide protection against HIV acquisition by reducing the risk of HSV-2 infection and its associated genital ulcers. However, the possibility of confounding because of sexual practices correlated with a high risk of transmission cannot be excluded. Two trials of HSV-2 suppression in HSV-2-positive, HIV-negative subjects did not show protection against HIV acquisition, ${ }^{38 \text {, }}$ 39 which suggests that more research is required on the acquisition of HSV-2 and HIV infections.

With respect to the use of circumcision to prevent HPV infection, our study was limited, since it was confined to a subgroup of subjects who were observed both at enrollment and at 24 months. In both the intervention and control groups, these subjects may represent a self-selected population of compliant subjects who could be at lower risk for HPV infection than the general population; this factor could result in an underestimation of the efficacy of male circumcision. Also, since samples were evaluated only at 24 months, we were limited in our ability to determine whether the reduced HPV prevalence after circumcision was due to a reduced rate 
of HPV acquisition, an increased rate of HPV clearance, or both. Nevertheless, these findings, in conjunction with data from observational studies 20,21 and one trial in South Africa, 31 demonstrate the efficacy of circumcision in decreasing the prevalence of HPV in adolescent boys and men.

Male circumcision has now been shown to decrease the rates of HIV, HSV-2, and HPV infections in men and of trichomoniasis and bacterial vaginosis in their female partners. ${ }^{7-9}$, 40 Circumcision also reduces symptomatic ulceration in HIV-negative men and women and HIV-positive men. $7,24,40$ Thus, male circumcision reduces the risk of several sexually transmitted infections in both sexes, and these benefits should guide public health policies for neonatal, adolescent, and adult male circumcision programs.

\section{Acknowledgements}

Supported by grants from the National Institutes of Health (U01-AI-51171), the Bill and Melinda Gates Foundation (22006.02), and the Fogarty International Center (5D43TW001508 and D43TW00015) and by the Intramural Research Program of the National Institute of Allergy and Infectious Diseases.

Dr. Gravitt reports receiving research funding from Roche Molecular Diagnostics, which manufactured the HPV genotyping test used in this study. No other potential conflict of interest relevant to this article was reported.

We thank Andrew Redd for his suggestions; Jordyn Gamiel, Jessica Neal, Roslyn Howard, and Lauren Wilson for their technical assistance; and the study participants and the Rakai Community Advisory Board, whose commitment and cooperation made this study possible.

\section{References}

1. Whitley RJ, Roizman B. Herpes simplex virus infections. Lancet 2001;357:1513-8. [PubMed: 11377626]

2. Corey L, Wald A, Celum CL, Quinn TC. The effects of herpes simplex virus-2 on HIV-1 acquisition and transmission: a review of two overlapping epidemics. J Acquir Immune Defic Syndr 2004;35:43545. [PubMed: 15021308]

3. Fenton KA, Breban R, Vardavas R, et al. Infectious syphilis in high-income settings in the 21st century. Lancet Infect Dis 2008;8:244-53. [PubMed: 18353265]

4. Serwadda D, Gray RH, Sewankambo NK, et al. Human immunodeficiency virus acquisition associated with genital ulcer disease and herpes simplex virus type 2 infection: a nested case-control study in Rakai, Uganda. J Infect Dis 2003;188:1492-7. [PubMed: 14624374]

5. de Sanjosé S, Diaz M, Castellsagué X, et al. Worldwide prevalence and genotype distribution of cervical human papilloma-virus DNA in women with normal cytology: a meta-analysis. Lancet Infect Dis 2007;7:453-9. [PubMed: 17597569]

6. Muñoz N, Bosch FX, de Sanjosé S, et al. Epidemiologic classification of human papillomavirus types associated with cervical cancer. N Engl J Med 2003;348:518-27. [PubMed: 12571259]

7. Gray RH, Kigozi G, Serwadda D, et al. Male circumcision for HIV prevention in men in Rakai, Uganda: a randomised trial. Lancet 2007;369:657-66. [PubMed: 17321311]

8. Bailey RC, Moses S, Parker CB, et al. Male circumcision for HIV prevention in young men in Kisumu, Kenya: a random-ised controlled trial. Lancet 2007;369:643-56. [PubMed: 17321310]

9. Auvert B, Taljaard D, Lagarde E, Sobngwi-Tambekou J, Sitta R, Puren A. Randomized, controlled intervention trial of male circumcision for reduction of HIV infection risk: the ANRS 1265 Trial. PLoS Med 2005;2(11):e298. [PubMed: 16231970][Erratum, PLoS Med 2006;3(5):e298.]

10. Auvert B, Buvé A, Lagarde E, et al. Male circumcision and HIV infection in four cities in sub-Saharan Africa. AIDS 2001;15(Suppl 4):S31-S40. [PubMed: 11686463]

11. Weiss HA, Buvé A, Robinson NJ, et al. The epidemiology of HSV-2 infection and its association with HIV infection in four urban African populations. AIDS 2001;15(Suppl 4):S97-S108. [PubMed: $11686471]$ 
12. Xu F, Markowitz LE, Sternberg MR, Aral SO. Prevalence of circumcision and herpes simplex virus type 2 infection in men in the United States: the National Health and Nutrition Examination Survey (NHANES), 1999-2004. Sex Transm Dis 2007;34:479-84. [PubMed: 17413536]

13. Lavreys L, Rakwar JP, Thompson ML, et al. Effect of circumcision on incidence of human immunodeficiency virus type 1 and other sexually transmitted diseases: a prospective cohort study of trucking company employees in Kenya. J Infect Dis 1999;180:330-6. [PubMed: 10395846]

14. Reynolds SJ, Shepherd ME, Risbud AR, et al. Male circumcision and risk of HIV-1 and other sexually transmitted infections in India. Lancet 2004;363:1039-40. [PubMed: 15051285]

15. Gray R, Azire J, Serwadda D, et al. Male circumcision and the risk of sexually transmitted infections and HIV in Rakai, Uganda. AIDS 2004;18:2428-30. [PubMed: 15622320]

16. Bwayo J, Plummer F, Omari M, et al. Human immunodeficiency virus infection in long-distance truck drivers in east Africa. Arch Intern Med 1994;154:1391-6. [PubMed: 8002691]

17. Cook LS, Koutsky LA, Holmes KK. Circumcision and sexually transmitted diseases. Am J Public Health 1994;84:197-201. [PubMed: 8296939]

18. Urassa M, Todd J, Boerma JT, Hayes R, Isingo R. Male circumcision and susceptibility to HIV infection among men in Tanzania. AIDS 1997;11:73-80.

19. Weiss HA, Thomas SL, Munabi SK, Hayes RJ. Male circumcision and risk of syphilis, chancroid, and genital herpes: a systematic review and meta-analysis. Sex Transm Infect 2006;82:101-9. [PubMed: 16581731]

20. Baldwin SB, Wallace DR, Papenfuss MR, et al. Human papillomavirus infection in men attending a sexually transmitted disease clinic. J Infect Dis 2003;187:1064-70. [PubMed: 12660920]

21. Castellsagué X, Bosch FX, Muñoz N, et al. Male circumcision, penile human papillomavirus infection, and cervical cancer in female partners. N Engl J Med 2002;346:1105-12. [PubMed: 11948269]

22. Shin HR, Franceschi S, Vaccarella S, et al. Prevalence and determinants of genital infection with papillomavirus, in female and male university students in Busan, South Korea. J Infect Dis 2004;190:468-76. [PubMed: 15243918]

23. Weaver BA, Feng Q, Holmes KK, et al. Evaluation of genital sites and sampling techniques for detection of human papillomavirus DNA in men. J Infect Dis 2004;189:677-85. [PubMed: 14767822]

24. Wawer, M.; Kigozi, G.; Serwadda, D., et al. Trial of male circumcision in HIV+ men, Rakai, Uganda: effects in HIV+ men and in women partners. Proceedings of the 15th Conference on Retroviruses and Opportunistic Infections; Boston. February 3-6; 2008. abstract

25. Gamiel JL, Tobian AAR, Laeyendecker OB, et al. Improved performance of enzyme-linked immunosorbent assays and the effect of human immunodeficiency virus coinfection on the serologic detection of herpes simplex virus type 2 in Rakai, Uganda. Clin Vaccine Immunol 2008;15:888-90. [PubMed: 18321879]

26. Gravitt PE, Peyton CL, Alessi TQ, et al. Improved amplification of genital human papillomaviruses. J Clin Microbiol 2000;38:357-61. [PubMed: 10618116]

27. Gravitt PE, Peyton CL, Apple RJ, Wheeler CM. Genotyping of 27 human papillomavirus types by using L1 consensus PCR products by a single-hybridization, reverse line blot detection method. J Clin Microbiol 1998;36:3020-7. [PubMed: 9738060]

28. Bosch FX, Manos MM, Muñoz N, et al. Prevalence of human papillomavirus in cervical cancer: a worldwide perspective. J Natl Cancer Inst 1995;87:796-802. [PubMed: 7791229]

29. Cogliano V, Baan R, Straif K, Grosse Y, Secretan B, El Ghissassi F. Carcinogenicity of human papillomaviruses. Lancet Oncol 2005;6:204. [PubMed: 15830458]

30. Sobngwi-Tambekou J, Taljaard D, Lissouba P, et al. Effect of HSV-2 serostatus on acquisition of HIV by young men: results of a longitudinal study in Orange Farm, South Africa. J Infect Dis 2009:199.(in press)

31. Auvert B, Sobngwi-Tambekou J, Cutler E, et al. Effect of male circumcision on the prevalence of high-risk human papillomavirus in young men: results of a randomized controlled trial conducted in Orange Farm, South Africa. J Infect Dis 2009;199:14-9. [PubMed: 19086814]

32. Szabo R, Short RV. How does male circumcision protect against HIV infection? BMJ 2000;320:15924. [PubMed: 10845974] 
33. McCoombe SG, Short RV. Potential HIV-1 target cells in the human penis. AIDS 2006;20:1491-5. [PubMed: 16847403]

34. Gupta R, Warren T, Wald A. Genital herpes. Lancet 2007;370:2127-37. [PubMed: 18156035]

35. Gray RH, Wawer MJ, Brookmeyer R, et al. Probability of HIV-1 transmission per coital act in monogamous, heterosexual, HIV-1-discordant couples in Rakai, Uganda. Lancet 2001;357:114953. [PubMed: 11323041]

36. Corey L, Adams HG, Brown ZA, Holmes KK. Genital herpes simplex virus infections: clinical manifestations, course, and complications. Ann Intern Med 1983;98:958-72. [PubMed: 6344712]

37. Corey L, Handsfield HH. Genital herpes and public health: addressing a global problem. JAMA 2000;283:791-4. [PubMed: 10683059]

38. Celum C, Wald A, Hughes J, et al. Effect of aciclovir on HIV-1 acquisition in herpes simplex virus 2 seropositive women and men who have sex with men: a ran-domised, double-blind, placebocontrolled trial. Lancet 2008;371:2109-19. [PubMed: 18572080]

39. Watson-Jones D, Weiss HA, Rusizoka M, et al. Effect of herpes simplex suppression on incidence of HIV among women in Tanzania. N Engl J Med 2008;358:1560-71. [PubMed: 18337596]

40. Gray RH, Kigozi G, Serwadda D, et al. The effects of male circumcision on female partners' genital tract symptoms and vaginal infections in a randomized trial in Rakai, Uganda. Am J Obstet Gynecol 2009;200(1):42.e1-42.e7. [PubMed: 18976733] 


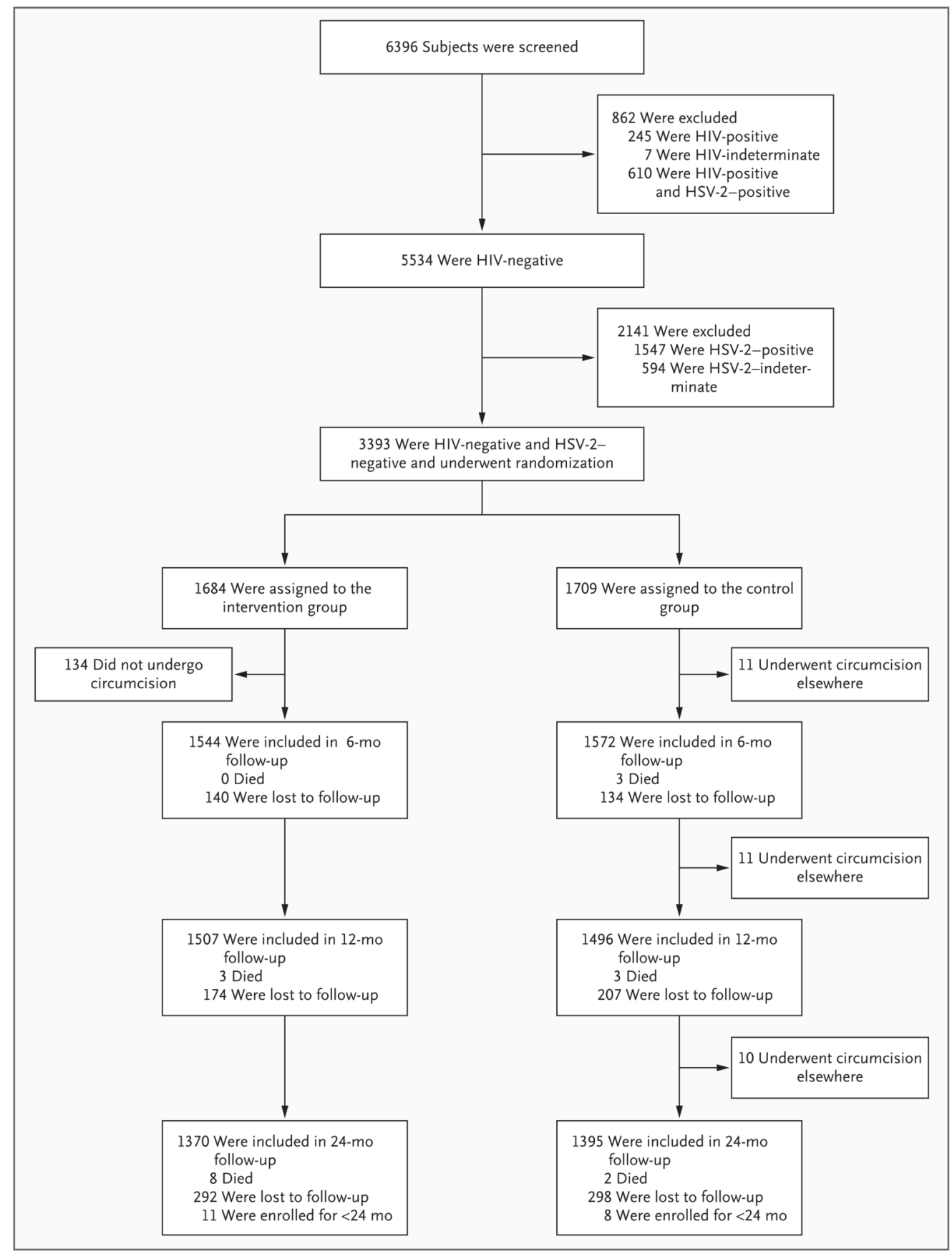

Figure 1. Enrollment and Outcomes in the Evaluation of Herpes Simplex Virus Type 2 (HSV-2) Infection

Some subjects were lost to follow-up at 6 months or 12 months but were then evaluated at 12 months or 24 months, so the numbers of subjects who were evaluated at each follow-up period do not necessarily tally with the numbers of subjects who were lost to follow-up for that period. All subjects who were seen during the trial are shown, but samples were not available for testing at month 6 for 13 subjects in the intervention group and 7 in the control group and at year 1 for 13 subjects in the intervention group and 8 in the control group. Not included in the 24month analyses were 11 subjects in the intervention group and 8 in the control group who had been enrolled in the trial for less than 24 months. 


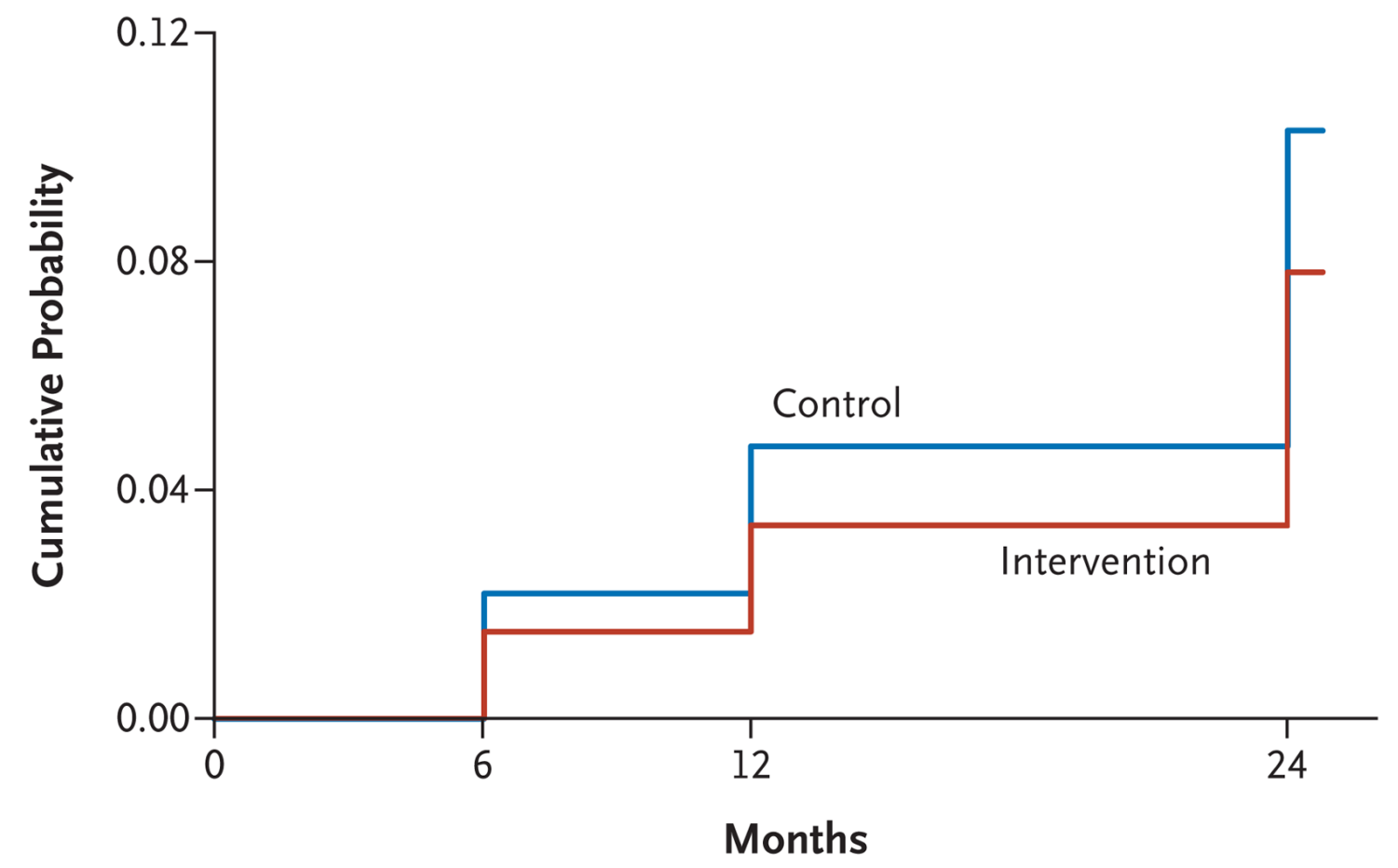

No. at Risk Intervention 1684

Figure 2. Kaplan-Meier Estimates of the Probability of Detection of Herpes Simplex Virus Type 2 (HSV-2) Infection at 24 Months

During the 24-month study period in the intention-to-treat population, HSV-2 infection was detected in 114 subjects who had undergone circumcision (intervention group) and in 153 subjects who had not undergone the procedure (control group). The cumulative probability of HSV-2 infection during the 24-month period was $7.8 \%$ in the intervention group, as compared with $10.3 \%$ in the control group, with an unadjusted hazard ratio of 0.75 (95\% CI, 0.60 to 0.94 ; $\mathrm{P}=0.02$ ). HSV -2 infection was initially detected at 6 months in 24 subjects in the intervention group and 35 in the control group and at 12 months in 29 subjects in the intervention group and 40 in the control group. At 24 months, HSV-2 infection was initially diagnosed in 61 subjects in the intervention group and 78 in the control group. 


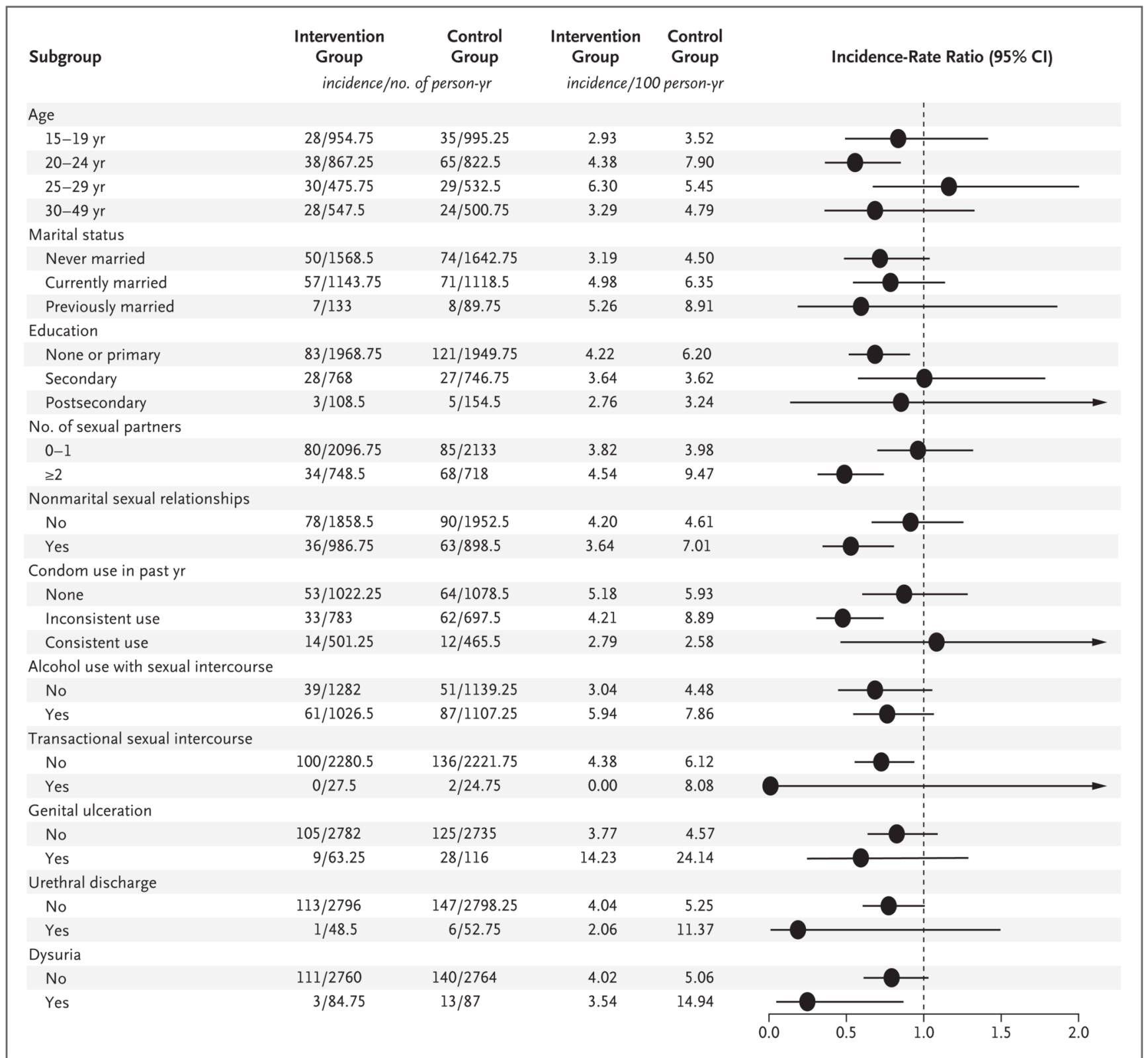

Figure 3. Incidence of Herpes Simplex Virus Type 2 (HSV-2) Infection and Incidence-Rate Ratios, According to Sociodemographic and Clinical Characteristics

Condom use, the use of alcohol with sexual intercourse, and transactional sexual intercourse were evaluated only in subjects who were sexually active. The horizontal lines represent $95 \%$ confidence intervals, with arrows indicating extensions of the intervals beyond 2.0. 


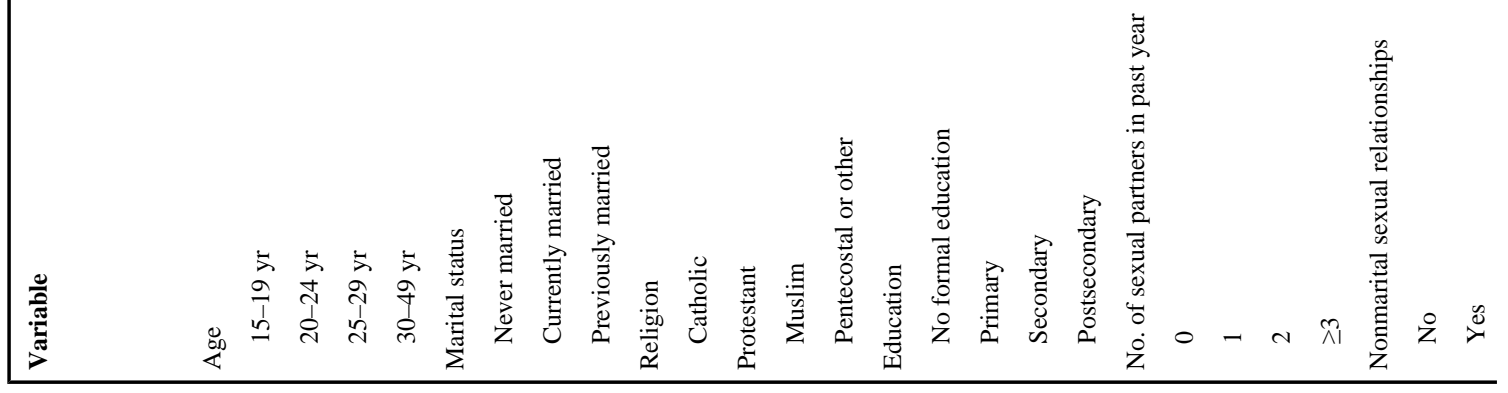




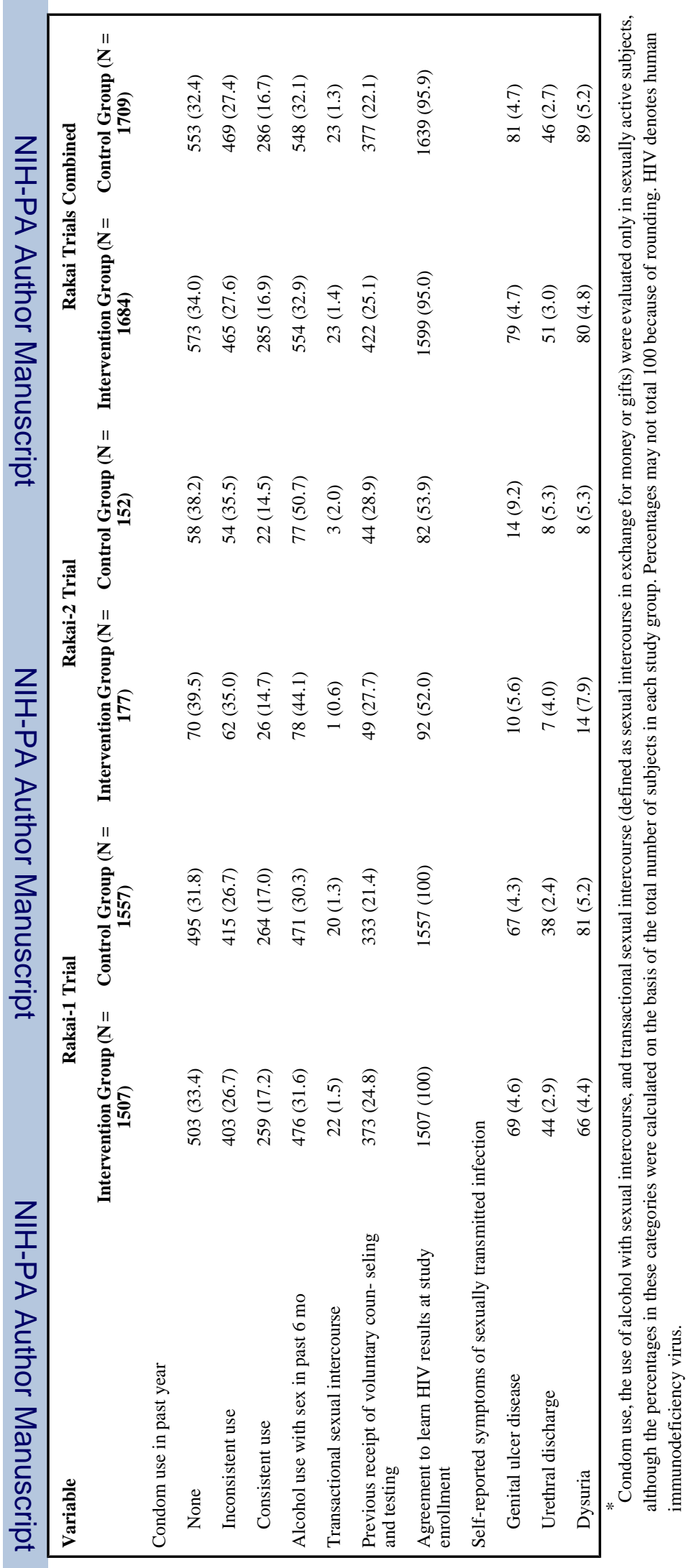

N Engl J Med. Author manuscript; available in PMC 2009 May 4. 
Table 2

Rates of Sexual Risk Behavior in the Evaluation of Herpes Simplex Virus Type 2 (HSV-2) Infection. *

\begin{tabular}{|c|c|c|c|}
\hline Variable & $\begin{array}{r}\text { Intervention Group } \\
\text { number }(p e\end{array}$ & $\begin{array}{l}\text { Control Group } \\
\text { ercent) }\end{array}$ & P Value \\
\hline \multicolumn{4}{|l|}{ At 6 mo } \\
\hline All subjects & $1544(100.0)$ & $1572(100.0)$ & \\
\hline No. of sexual partners in past yr & & & 0.19 \\
\hline 0 & $377(24.4)$ & $434(27.6)$ & \\
\hline 1 & $831(53.8)$ & $816(51.9)$ & \\
\hline 2 & $247(16.0)$ & $228(14.5)$ & \\
\hline$\geq 3$ & $89(5.8)$ & $94(6.0)$ & \\
\hline Nonmarital sexual relationships & $514(33.3)$ & $507(32.3)$ & 0.54 \\
\hline Condom use & & & $<0.001$ \\
\hline None & $463(39.7)$ & $543(47.7)$ & \\
\hline Inconsistent use & $422(36.2)$ & $347(30.5)$ & \\
\hline Consistent use & $282(24.2)$ & $248(21.8)$ & \\
\hline Alcohol use with sexual intercourse & $503(43.1)$ & $547(48.1)$ & 0.02 \\
\hline \multicolumn{4}{|c|}{ Self-reported symptoms of sexually transmitted infection } \\
\hline Genital ulcer disease & $24(1.6)$ & $60(3.8)$ & $<0.001$ \\
\hline Urethral discharge & $20(1.3)$ & $23(1.5)$ & 0.69 \\
\hline Dysuria & $37(2.4)$ & $39(2.5)$ & 0.88 \\
\hline \multicolumn{4}{|l|}{ At $12 \mathrm{mo}$} \\
\hline All subjects & $1507(100.0)$ & $1496(100.0)$ & \\
\hline No. of sexual partners in past yr & & & 0.33 \\
\hline 0 & $346(23.0)$ & $386(25.8)$ & \\
\hline 1 & $808(53.6)$ & $775(51.8)$ & \\
\hline 2 & $285(18.9)$ & $274(18.3)$ & \\
\hline$\geq 3$ & $68(4.5)$ & $61(4.1)$ & \\
\hline Nonmarital sexual relationships & $536(35.6)$ & $478(32.0)$ & 0.04 \\
\hline Condom use & & & 0.95 \\
\hline None & $547(47.1)$ & $527(47.5)$ & \\
\hline Inconsistent use & $352(30.3)$ & $330(29.7)$ & \\
\hline Consistent use & $262(22.6)$ & $253(22.8)$ & \\
\hline Alcohol use with sexual intercourse & $534(46.0)$ & $557(50.2)$ & 0.05 \\
\hline \multicolumn{4}{|c|}{ Self-reported symptoms of sexually transmitted infection } \\
\hline Genital ulcer disease & $26(1.7)$ & $64(4.3)$ & $<0.001$ \\
\hline Urethral discharge & $19(1.3)$ & $22(1.5)$ & 0.54 \\
\hline Dysuria & $33(2.2)$ & $38(2.5)$ & 0.43 \\
\hline \multicolumn{4}{|l|}{ At 24 mo } \\
\hline All subjects & $1370(100.0)$ & $1395(100.0)$ & \\
\hline No. of sexual partners in past yr & & & 0.46 \\
\hline 0 & $200(14.6)$ & $224(16.1)$ & \\
\hline 1 & $719(52.5)$ & $722(51.8)$ & \\
\hline 2 & $314(22.9)$ & $329(23.6)$ & \\
\hline$\geq 3$ & $137(10.0)$ & $120(8.6)$ & \\
\hline
\end{tabular}




\begin{tabular}{|lccc|}
\hline Variable & Intervention Group & Control Group & P Value \\
Nonmarital sexual relationships & $503(36.7)$ & $458(32.8)$ & 0.03 \\
Condom use & & & 0.12 \\
$\quad$ None & $471(40.5)$ & $520(44.7)$ & \\
Inconsistent use & $443(38.1)$ & $415(35.7)$ & \\
Consistent use & $249(21.4)$ & $228(19.6)$ & \\
Alcohol use with sexual intercourse & $586(50.4)$ & $647(55.6)$ & 0.01 \\
Self-reported symptoms of sexually transmitted infection & & \\
$\quad$ Genital ulcer disease & $48(3.5)$ & $75(5.4)$ & 0.02 \\
$\quad$ Urethral discharge & $31(2.3)$ & $39(2.8)$ & 0.37 \\
Dysuria & $53(3.9)$ & $60(4.3)$ & 0.57 \\
\hline
\end{tabular}

Condom use and the use of alcohol with sexual intercourse were evaluated only in sexually active subjects. In the intervention group, those numbers were 1167 at 6 months, 1161 at 12 months, and 1163 at 24 months; in the control group, those numbers were 1138 at 6 months, 1110 at 12 months, and 1163 at 24 months. At 24 months, data were missing for seven subjects in the intervention group and eight in the control group. 
Table 3

Male Circumcision and the Prevalence of Human Papillomavirus (HPV) Infection.*

\begin{tabular}{|lccc|}
\hline HPV Genotype & HPV-Positive & Risk Ratio (95\% CI) \\
& $\begin{array}{c}\text { Intervention Group Control Group } \\
\text { no./total no. (\%) }\end{array}$ \\
All genotypes & & & \\
At baseline & $190 / 307(61.9)$ & $189 / 302(62.6)$ & $0.99(0.81-1.21)$ \\
At 24 mo & $83 / 233(35.6)$ & $147 / 287(51.2)$ & $0.70(0.53-0.91)$ \\
Low-risk genotypes & & & \\
At baseline & $146 / 307(47.6)$ & $145 / 302(48.0)$ & $0.99(0.79-1.25)$ \\
At 24 mo & $61 / 233(26.2)$ & $113 / 287(39.4)$ & $0.66(0.49-0.91)$ \\
High-risk genotypes & & & \\
At baseline & $117 / 307(38.1)$ & $112 / 302(37.1)$ & $1.03(0.79-1.33)$ \\
At 24 mo & $42 / 233(18.0)$ & $80 / 287(27.9)$ & $0.65(0.45-0.94)$ \\
\hline
\end{tabular}

* The total number of subjects includes all those who had detectable beta-globin or HPV. Subjects were included in both the high-risk category and the low-risk category if they had both HPV genotypes. CI denotes confidence interval. 